\title{
Effectiveness of Mobile Apps Used to Improve Urinary Incontinence: A Systematic Review of Randomised Controlled Trials
}

\section{Renee Widdison}

Edith Cowan University

Amineh Rashidi ( $\sim$ a.rashidi@ecu.edu.au )

Edith Cowan University

Lisa Whitehead

Edith Cowan University

\section{Research Article}

Keywords: m-Health, mobile applications, mobile apps, pelvic floor muscle training, adherence, urological

Posted Date: August 11th, 2021

DOI: https://doi.org/10.21203/rs.3.rs-620368/v1

License: (1) This work is licensed under a Creative Commons Attribution 4.0 International License.

Read Full License

Version of Record: A version of this preprint was published at BMC Nursing on January 28th, 2022. See the published version at https://doi.org/10.1186/s12912-022-00812-6. 


\section{Abstract}

Introduction: Pelvic floor exercises are effective in the treatment of urinary incontinence (UC) and are routinely prescribed, along with bladder training, by primary healthcare providers as first line conservative management. Mobile phone applications are increasingly popular within the healthcare setting and can provide opportunities for patients to complete treatments at home. To date, there has not been a systematic review examining outcomes from randomised controlled trials on the effectiveness of mobile applications to improve UC.

Methods: A systematic review of randomized controlled trials evaluating the effectiveness of mobile applications to improve UC was carried out according to the PRISMA reporting guidelines. The online databases MEDLINE, Embase, PsychINFO, CINAHL, Web of Science, Scopus, The Cochrane Library, JBI, Google Scholar were searched for papers published between 2007 to 2020. The search was conducted during the $3 / 3 / 20-15 / 4 / 20$. Studies were appraised and data extracted by the first and second reviewers.

Findings: In total four studies generated four categories, including high level of adherence; improved quality of life (QOL) and reduction of symptoms. The findings of this review reported that pelvic floor muscle training (PFMT) can be effective to improve UC.

Conclusion: Mobile applications for PFMT indicated that increase adherence to treatment and decrease UC. The integration of this treatment modality into current practice is recommended. Mobile phone applications for PFMT show promise in the conservative management of UC. Further research is required to support the use of this technology in the conservative management of UC.

\section{Background}

An estimated 400 million adults worldwide experience urinary incontinence (UC) (1). Pelvic floor muscle training (PFMT) is a conservative first line treatment for UC defined as the involuntary loss of urine (2). There is evidence emerging from randomized controlled trials signifying improvement of UC and quality of life (QOL) by adherence to pelvic floor training. Pelvic floor muscles are like a funnel and contain the Levator ani muscles and further divided into the Puborectalis, Pubococcygeus, lliococcygeus, alongside the Coccygeus muscle and supporting fascia. These muscles support the visceral organs including the bladder, urethra, uterus, and bowel and are responsible for maintaining continence (McAninch \& Lue,2019). Pelvic floor muscle weakness is as a disorder affecting both men and women which contributes to UC. Conditions contributing to weak pelvic floor muscles and UC include obesity, constipation, pregnancy, childbirth, menopause, heavy lifting, surgery, trauma, and radiation treatment (The Continence Foundation Australia, 2020). Strengthening the pelvic floor muscles can be achieved through a set of exercises specifically targeting muscle groups.

Currently, pelvic floor strength and contraction duration remain important measurements in pelvic floor assessment. PFMT exercises offer conservative management for UC and are routinely prescribed for treatment of UC, in conjunction with improved fluid intake management and bladder habits. Patients are 
required to complete exercises daily at home as part of their treatment plan. Recommended guidelines with high evidence of effectiveness exist in current practice (3)Health care professionals have access to these guidelines however they do not always follow them, and locally developed programs exist instead (3). The traditional method of face to face training disadvantages some people from participation in training programs. Uptake to training programs is notably affected by service provision and challenges accessing services (Salmon et al, 2019). PFMT delivered via mobile applications are easily accessed by people in the home setting and have been found to be advantageous in overcoming known barriers to training whilst also offering the same outcomes as stand-alone practice (4). Recent studies indicate that mobile applications for the treatment of UC can be of value in future treatment (4). Mobile applications have been reported as user friendly, with the capability of saving favourite exercise routines and can act as reminder for personalised exercise (4). The functions within the mobile application enabled and encouraged participants, who have saved the correct number of repetitions and obtained optimal results and satisfaction (4). There is yet to be a systematic review of the effectiveness of mobile application used to improve UC. Therefore, this review extends the current literature providing a narrative synthesis of the most recent randomised controlled trials evaluating mobile applications to deliver PFMT for UC.

\section{Aim:}

This review aimed to synthesize evidence from randomised controlled trials to examine the effectiveness of mobile applications delivering PFMT to improve UC.

\section{Methods}

\subsection{Search strategy}

The searches were conducted in MEDLINE, Embase, PsychINFO, CINAHL, Web of Science, Scopus, The Cochrane Library, Joanna Briggs Institute JBI, and Google Scholar. The search was performed using the following key search terms: pelvic floor exercise*, mobile app*, Kegel exercise*, mobile application* Studies in the English language, peer-reviewed studies between 2007 and 2020, with patient populations of adults over 18 years and diagnosis of UC were included. This review also considered studies focused on the uptake of mobile phone applications to improve pelvic floor exercise. Studies with paediatric or adolescent UC, and prior to 2007 were also excluded as mobile phone applications were not available. Initially, $(n=416)$ articles were identified and $n=270$ articles were removed as duplicates (Appendix 1). First and second reviewers performed title and abstract screening, then they conducted full text screening (5).

\subsection{Quality appraisal and data extraction}


Studies were appraised by the all reviewers, using the standard JBI Critical Appraisal Checklist for Randomised Controlled trials (5). There were no disagreements between the reviewers. Using the JBI data extraction tool for data extraction, including specific details about the sample, setting, location, data collection and data analysis and relevant to the review objective; examining the effectiveness of mobile applications used to improve UC.

\subsection{Data Synthesis}

The randomised controlled trials included in the review were heterogenous in relation to their treatment protocols and a meta-analysis could not be completed. A narrative synthesis was conducted involving the aggregation and synthesis.

\section{Results}

\subsection{Study Inclusion}

The published studies were between 2016 and 2020 and were undertaken in Sweden, Canada, Brazil, and China. Of the four trials, three were complete trials, and one a two-year follow-up post completion of an RCT. A variety of primary and secondary outcomes were assessed across the trials. Outcomes documented in the trials included symptom severity, QOL and adherence to PFMT, bladder neck function, sexual activity, cure rates and use of incontinence products. Three studies (6-8) included symptom severity and adherence as primary outcomes. One study (9) continued with the same outcomes from the Asklund, Nyström (7) trial as they conducted the two year follow up. Each study clearly defined the primary and secondary outcomes and time to follow-up varied across the studies.

\subsection{Quality of included studies}

The JBI quality appraisal tool, a 13-point checklist for randomised controlled trials was used to assess the quality of the four studies (Appendix 2). The methodological quality of the studies was assessed based on methods of randomisation, allocation concealment, blinding, outcomes, statistical analysis, and loss to follow-up. True randomisation, allocation concealment and similar baseline were unanimous across the studies except for the two-year follow-up trial. Blinding was true amongst all participants in the studies conducted by two studies $(6,7)$. Blinding for participants was declared not feasible by two studies $(8,9)$. Those delivering treatments were blinded in only one study $(8)$. True blinding of outcome assessors did not occur in the four studies.

\subsection{Review Findings}


The findings synthesised into three categories, appendix 3 presents an overview of the study characteristics.

\section{Adherence to PFMT using a mobile application and perceived value of using a mobile application}

Four studies generated this findings (6-9). These four studies determined the success of adherence to PFMT using a mobile application by evaluating self-reporting measures, urinary symptoms, app utilisation and self-efficacy. The adherence rate was higher in the mobile application group and this was evident after one-two months of pelvic floor exercise, reflected in $\mathrm{P}<0.001$. Self-reported adherence in the mobile group showed better results during treatment, also reflected by $\mathrm{P}<0.001$ (6). Asklund et al (2016) found a higher adherence rate in the mobile application group, they report that $41 \%$ performed pelvic floor exercises daily and this was vastly different to the control group who recorded a $3.3 \%$ adherence rate to daily pelvic floor exercise. Hoffman et al (2017) followed up the original Asklund et al (2016) study and of the 46 women who participated in this follow-up, all 46 women had downloaded and kept the mobile application and $17.4 \%$ were still using it two years later.

Frequent users who intend to use mobile applications on a regular basis, indicated higher PFMT adherence compared to those performing stand-alone exercises... "based on our results women who used it not only exercised more than the other group, based on exercise sections duration but also felt more committed to exercising"( Arajou et al., 2019; p.6). Also, people who used mobile applications in the short term were satisfied about the outcome: "with the present study we have shown the app treatment to be effective in the short term" (Asklund et al., 2016; p.1375). The high-level adherence rate of PFMT using an application resulted in participants suffering less from pelvic floor injury, less bladder neck descent and improved pelvic floor strength: "participants receiving the app-based audio guidance indicated more positive effect to 6 months postpartum" (Wang et al.,2020; p. 7). Patients who used mobile applications to undertake exercise reported positive aspects of the application: "the reminder notes were important to exercise adherence and preferred to continue using the said feature" (Arajou et al., 2019; p.6).

\section{QOL}

Findings from three studies, Arajou et al (2019), Asklund et al (2016) and Hoffman et al (2017) contributed to this synthesised finding. QOL measures are an estimation of wellbeing which can be assessed by measuring improvements in areas like healthcare and are based on an individual's perception and expectations of treatment outcomes. In the app group Asklund et al (2016) presented a mean score reduction in QOL scores 4.8, 95\% Cl: 3.4-6.2 and the control group did not have a mean score reduction in QOL. QOL was measured among other outcomes and the app group did show improvement with $P$ values $=0.003$ Araujo et al (2019). It is well established that the physical, psychological, and sociological effects of UC affect QOL amongst diverse patient groups and mobile applications easily 
provide access to life changing treatments with recurrent users ... "experiencing fewer symptoms and reporting a better QOL" (Asklund et al., 2016; p.1373). Discovering long term benefits of PFMT using an application has improved QOL in mobile users has been a success in long term studies ... "our study yielded promising long-term results in terms of a condition that can severely affect QOL" (Hoffman et al., 2017; p.1185).

\section{Symptom Improvement}

Findings from four studies (Arajou et al (2019), Asklund et al (2016), Hoffman et al (2017) \& Wang et al (2020) contribute the synthesised finding of reduction in symptoms. To benefit from a treatment program delivered by a mobile application is outstanding and has been acknowledged as a successful outcome in these recent studies, with participants reporting positive results using an application for PFMT to reduce UC. Symptom reduction measured by validated assessment tools and methods provides valuable data and true insight into the condition "people experience greater improvement in symptom severity with a mean reduction in ICIQ scores" (Asklund et al., 2016; p.1372) and "we found that PFMT improves symptoms of UC, according to validated questionnaires" (Arajou et al., 2019; p.6). Furthermore, the cost and burden associated with continence aids is high and patients frequently ask during consultation when they will be able to cease using pads (Coyne et al 2014). "Outcome measurements at follow-up determined $56 \%$ of the app group as no leakage or $>50 \%$ fewer leakage episodes than at baseline" (Asklund et al., 2016; p.1372). Physically, a strong pelvic floor can improve and maintain continence as well as delay surgical intervention and the associated risks of major surgery (Giarenis \& Robinson, 2014). Pelvic floor strength and less bladder neck descent has been noted in frequent users of mobile applications for pelvic floor muscle training: "participants had significant improvements in symptom severity after 3 months" (Wang et al., 2020; p.5), reiterating the practicality of an accessible PFMT program and the importance of conservative management of UC.

\section{Discussion}

The purpose of this review was to synthesise the evidence of the effectiveness of mobile applications used to improve UC. Four randomised controlled trials were included, and all four randomised controlled trials were considered high quality according to the JBI randomised controlled trial critical appraisal checklist (5). The heterogeneity of the included studies determined a narrative synthesis appropriate. This review yielded 87 findings that led to the generation of four categories and three syntheses. Adherence to PFMT was key to effective treatment outcomes (6-9). The success and effectiveness of treatment outcomes using a mobile application is attributed to adherence and a mobile application provides flexibility enabling the patient to take control and manage their condition at home. For example, a relatively new and attractive treatment modality provides reminder functions, feedback data, audio guidance and most importantly privacy. High level adherence had a direct impact on symptom reduction and improved QOL and these outcomes are of particular interest to health care providers aiming to provide results to their patients. Symptom reduction and improved QOL is a shared goal for both the 
provider and patient. (10). Such integration would require health promotion focussing on the established benefits and emphasising high level adherence to PFMT is achievable with a mobile application (11).

Mobile applications provide an alternative to regular pelvic floor muscle training, reduce symptoms and particularly provide opportunity to overcome health care barriers. Recent evidence indicated that PFMT is as an effective treatment to reduce UC (10). Likewise, the advantages of using a mobile application for treatment and users adhered to PFMT long enough for physiological change to occur within the Levator ani, Puborectalis, Pubococcygeus, lliococcygeus, Coccygeus muscles and supporting fascia and consequently improve QOL (12).

Physiological outcome measures provide the strongest evidence of a mobile application delivering PFMT for a reduction in UC as effective. Results signify that increased pelvic floor muscle strength maintained bladder neck support, decreased bladder and urethral pressure maintaining urethral closure and therefore preventing UC, as a direct result of adherence to PFMT via a mobile application (6-9). Equally as burdensome are the physiological effects of UC and the direct impact on QOL (Mallah et al.,2014). The financial burden of outlaying costs for a never-ending supply of continence products can escalate individual stress levels, as dose the personal shame of living with UC. It is well documented that barriers to health care are detrimental to good outcomes and leaving the offending condition untreated greatly impacts QOL (Australian Institute of Health and Welfare, 2013). Pleasingly the results from (6-9) have proven an alternative method to deliver PFMT proving it can overcome barriers and provide the same results as health care led pelvic floor muscle training. The flexibility of a mobile application empowers the patient in their own care, adherence is ensured, and the desired effects of symptom reduction and improved QOL are achieved and all by the touch of a finger.

\section{Limitation}

This review focussed solely on women experiencing UC and there is indication for further research into this area, expanding on the limitations of the earlier studies. Other limitations in general for example, paediatric population, males, non-English studies, or developing countries. This systematic review focussed on randomised controlled trials, the limitation being the small number of trials available. The literature search for this systematic review did not identify any qualitative studies examining in this area of research.

\section{Conclusions}

The effectiveness of mobile applications used to improve UC has been established within the studies included in this review. Adherence is key to PFMT and mobile applications provide flexible training in the home overcoming healthcare barriers. Mobile applications guiding PFMT increase adherence to treatment and reduce UC therefore improving QOL. Future research will contribute to and guide clinical practice. 


\section{Declarations}

\section{Ethics approval and consent to participate}

The review was approved by the Edith Cowan University Human Research Ethics Committee (202001499). There was no requirement to obtain consent from participants as all data generated or analysed during this study are included in this published article.

\section{Consent for publication}

Not applicable

\section{Availability of data and materials}

All data generated or analysed during this study are included in this published article [and its supplementary information file 2]. Datasets are available through the first author upon reasonable request.

\section{Competing interests}

The authors declare that they have no competing interest

\section{Funding}

Not applicable

\section{Authors' contributions}

The author RW conceived the review and oversight for all stages of the review provided by AR and LW. RW and AR undertook the initial database search, data extraction was undertaken by RW. All authors conducted screening and critical appraisal. RW analysed the data and wrote the first draft of the manuscript. All authors reviewed, revised the manuscript, and approved the final version of the manuscript.

\section{Acknowledgements}

Not applicable 


\section{References}

1. Hunter KF, Wagg AS. Improving nurse engagement in continence care. Nursing: Research and Reviews. 2018;8:1-7.

2. Nyström E, Asklund I, Sjöström M, Stenlund H, Samuelsson E. Treatment of stress urinary incontinence with a mobile app: factors associated with success. International urogynecology journal 2018;29(9):1325-33.

3. Venegas M, Carrasco B, Casas-Cordero R. Factors influencing long-term adherence to pelvic floor exercises in women with urinary incontinence. Neurourology and urodynamics 2017;36(5):1369-76.

4. Asklund I, Samuelsson E, Hamberg K, Umefjord G, Sjöström M. User experience of an app-based treatment for stress urinary incontience: a qualitative interview study. Journal of medical Internet research 2019;21(3): e11296.

5. Joanna Briggs Institute. Joanna Briggs Institute. System for the unified management, assessment and review of information, software for the systematic review of literature (JBI SUMARI). Retrieved from http://joannabriggs.org/. 2018. 2018.

6. Araujo CC, Marques AdA, Juliato CR. The adherence of home pelvic floor muscles training using a mobile device application for women with urinary incontinence: A randomized controlled trial. Female Pelvic Medicine and Reconstructive Surgery. 2020;26(11):697-703.

7. Asklund I, Nyström E, Sjöström M, Umefjord G, Stenlund H, Samuelsson E. Mobile app for treatment of stress urinary incontinence: A randomized controlled trial. Neurourology and urodynamics 36, no 5 (2017): 1369-1376. 2017;36(5):1369-76.

8. Wang X, Xu X, Luo J, Chen Z, Feng S. Effect of app-based audio guidance pelvic floor muscle training on treatment of stress urinary incontinence in primiparas: a randomized controlled trial. International journal of nursing studies. 2020;104:103527.

9. Hoffman V, Söderström L, Samuelsson E. Self-management of stress urinary incontinence via a mobile app: two-year follow-up of a randomized controlled trial. Acta obstetricia et gynecologica Scandinavica 2017;96(10):1180-7.

10. Wiegersma M, Panman C, Kollen BJ, Berger MY, Leeuwen YL-V, Dekker JH. Effect of pelvic floor muscle training compared with watchful waiting in older women with symptomatic mild pelvic organ prolapse: randomised controlled trial in primary care. BMJ. 2014;349.

11. Sacristán JA. Clinical research and medical care: towards effective and complete integration. BMC Medical Research Methodology. 2015;15(1):1-7.

12. Pereira-Baldon VS, Avila MA, Dalarmi CB, Oliveira ABd, Driusso P. Effects of different regimens for pelvic floor muscle training in young continent women: Randomized controlled clinical trial Journal of Electromyography and Kinesiology. 2019;44:31-5.

\section{Supplementary Files}


This is a list of supplementary files associated with this preprint. Click to download.

- Appendices.Supplementaryfile1..docx

- AvailabilityData.Supplementaryfile2.docx

- PRISMA2020checklist1.docx 